\title{
Improving hand hygiene in hospitals: comparing the effect of a nudge and a boost on protocol compliance
}

\author{
Henrico van Roekel$^{\star}$ (D), Joanne Reinhard and Stephan Grimmelikhuijsen \\ Utrecht School of Governance, Utrecht University, Utrecht, The Netherlands, \&samhoud Consultancy, \\ Utrecht, The Netherlands and Utrecht School of Governance, Utrecht University, Utrecht, The Netherlands \\ ${ }^{\star}$ Correspondence to: Utrecht School of Governance, Utrecht University, Bijlhouwerstraat 6-8, 3511 ZC \\ Utrecht, The Netherlands. E-mail: h.vanroekel@uu.nl
}

(Received 13 August 2020; revised 2 March 2021; accepted 10 March 2021; first published online 3 May 2021)

\begin{abstract}
Nudging has become a well-known policy practice. Recently, 'boosting' has been suggested as an alternative to nudging. In contrast to nudges, boosts aim to empower individuals to exert their own agency to make decisions. This article is one of the first to compare a nudging and a boosting intervention, and it does so in a critical field setting: hand hygiene compliance of hospital nurses. During a 4-week quasi-experiment, we tested the effect of a reframing nudge and a risk literacy boost on hand hygiene compliance in three hospital wards. The results show that nudging and boosting were both effective interventions to improve hand hygiene compliance. A tentative finding is that, while the nudge had a stronger immediate effect, the boost effect remained stable for a week, even after the removal of the intervention. We conclude that, besides nudging, researchers and policymakers may consider boosting when they seek to implement or test behavioral interventions in domains such as healthcare.
\end{abstract}

Keywords: boosting; healthcare; quasi-experiment; nudging; hand hygiene

\section{Practitioners' points}

- Both a reframing nudge and a risk literacy boost are shown to be effective behavioral interventions to improve hand hygiene in a critical healthcare setting.

- The effect of the boosting intervention remained stable for a week, even after the intervention was removed.

- In cases where nudging has considerable drawbacks, boosting can be a viable alternative when designing behavioral interventions.

\section{Introduction}

Nudging has become an increasingly accepted and successful policy instrument to steer the behavior of citizens and public professionals. Nudges are subtle interventions that 
alter the decision environment, aimed to help individuals make better decisions (Thaler \& Sunstein, 2008). Nudges do not require much cognitive or motivational effort on the side of the receiver of the nudge, which is often seen as a core selling point in contexts where individuals have little time to reflect on the 'best' decision (e.g., Münscher et al., 2016). At the same time, the ethics and effectiveness of nudging have been subject to debate. Some raise moral objections, such as the reduced individual autonomy (e.g., Wilkinson, 2013), while others emphasize the short-term duration of nudges: the effect of a nudge may whither, and over time, existing cognitive biases may prevail again (Hertwig, 2017).

In response, a different kind of behavioral intervention known as 'boosting' is proposed as another option to change behavior in a similarly cost-effective way, but with some differences. In contrast to nudging, boosting aims to improve the decision process of the individual and presents a more reflective counterpart to nudging (Grüne-Yanoff et al., 2018; Lorenz-Spreen et al., 2020). For instance, a boost can help eliminate flaws in decision-making by training an individual's math skills. Boosting is considered to change behavior not by changing the choice environment but by empowering individuals and strengthening their competence (Hertwig \& Grüne-Yanoff, 2017).

Boosts do not necessarily impose high costs for policymakers. For instance, medical doctors' understanding of health statistics increases greatly when such statistics are presented in a frequency, as opposed to probabilities (e.g., Hoffrage et al., 2000). At the same time, other boost-related interventions may require more time investment. For instance, entrepreneurs' financial decision-making skills can be trained by teaching them simple financial and accounting heuristics (Drexler et al., 2014).

In many cases, both nudging and boosting approaches can potentially be applied, yet there are no empirical studies systematically comparing the effectiveness of a nudge and a boost in healthcare. Testing these interventions in a high-stakes hospital context is highly relevant for three reasons.

First, the importance of hand hygiene to prevent infections needs little explanation since the COVID-19 crisis. That said, hand hygiene compliance has always been crucial in hospitals to halt hospital-induced infections. All hospitals have standardized protocols in place that provide details about when and how hand hygiene should be applied. However, many studies report compliance rates under 50\% (e.g., Squires et al., 2013), which causes infections to be transmitted between patients and hospital employees (Pittet et al., 2006). Consequentially, 1 in every 20 patients in (Dutch) hospitals suffers from hospital-induced infections (IGJ, 2018) and 20.4\% of all hospital deaths were at least partly caused by hospital-induced infections (Langelaan, 2017).

Secondly, improving hand hygiene using behavioral interventions in a hospital context is relevant from a policy perspective, too. Top-down approaches by policymakers such as bans and mandates may cause feelings of job alienation (Tummers et al., 2009). Scholars in public administration show that public professionals such as nurses work in demanding contexts with increasing needs for accountability and administration (Noordegraaf, 2007). In such demanding environments, behavioral interventions such as nudging and boosting may help alter behavior in an effective way without alienating them from their job.

Thirdly, by comparing a boost and nudge intervention in a hospital setting, we test existing behavioral insights on motivational biases. Nurses and doctors tend to be 
overconfident about their personal immunity (e.g., Klitzman, 2006). Such overconfidence may be driven by motivational biases, such as motivated reasoning (Kunda, 1990). For instance, hospital staff may need to convince themselves that they are immune to disease to feel secure during their work. Interventions that frame hand hygiene to improve the nurses' health may suffer from confirmation bias and, therefore, not be effective (Nickerson, 1998). Instead, it is suggested that interventions in hand hygiene should focus on the consequences on others (Grant \& Hofmann, 2011).

Based on these insights, this study develops and tests a nudge and boost intervention in a hospital. The nudge reframes hand hygiene as a moment of care for the patient, shifting the beneficiary of hand hygiene toward the patient rather than the nurse or doctor. The boost focuses on improving risk literacy - a common type of boost - and doing so, it shows nurses that hand hygiene results in severe risks for patients. We test both interventions and compare their effectiveness.

This leads to the following research question: What is the effect of nudging and boosting on hand hygiene compliance of nurses? This question was tested in a 4 -week nonrandomized quasi-experiment in three wards in a medium-large Dutch hospital, executed in April-May 2019. The interventions were implemented at the ward level. We tested a boosting-oriented intervention in one ward, a nudge in another ward, and included a control group with no intervention (business as usual).

Quasi-experimentation is a valuable method in assessing causality when fully randomized designs are not possible or desirable (Shadish et al., 2002; Grant \& Wall, 2009). We took several measures to support causal identification: we included multiple pre- and post-tests for each ward, and we included a control group and used objective measures of hand hygiene behavior (Shadish et al., 2002). To further validate our design, the three wards had been selected for a number of similarities: they all provide nonintensive care to a large variety of patients and have similar ward and personnel structure.

Overall, we make two main contributions. First, we contribute to the field of behavioral public administration and policy (Grimmelikhuijsen et al., 2017; Sunstein, 2020). A lot of scholarly attention in this field has been devoted to either identifying cognitive biases among public professionals (e.g., Bellé et al., 2018) and citizens (e.g., Olsen, 2015) or applying nudging techniques that make the use of these biases to steer them in the 'right' direction (e.g., Andersen \& Hvidman, 2020). By showing the potential of boosting, specifically a risk literacy boost (Hertwig \& Grüne-Yanoff, 2017), we enrich the toolkit of possible behavioral interventions to improve public professional performance. By going beyond nudging, we explore boosting as an approach that encourages public professionals to change their behavior by exerting their own agency.

Our second contribution is to the rising number of studies on nudging interventions. There are no 'one-size-fits-all' nudges, and contextual differences are important in determining the effect of nudges (Jones, 2017; Sunstein, 2017). Given the highstakes professional context, it is not surprising that many studies have already applied some types of nudging intervention in the healthcare domain. A recent literature review by Nagtegaal et al. (2019) shows that many of these studies suffer from methodological shortcomings and tend to ignore certain types of nudges. In response, this article presents original evidence from a preregistered quasi-experiment using a little- 
tested nudge in this setting: reframing information. By showing that this type of nudge is effective, we potentially have evidence for a simple tool that helps improve hand hygiene compliance.

\section{Nudging versus boosting}

Before we discuss the potential effects of nudging and boosting on public professionals, we define both concepts and delineate their core differences. Nudging is traditionally concerned with influencing 'people's behavior in a predictable way', yet 'without forbidding any options or significantly changing their economic incentives' (Thaler \& Sunstein, 2008, p. 6). Nudging is founded on widely supported psychological findings on the existence of biases and flaws in the bounded rational human decisionmaking (Tversky \& Kahneman, 1981; Battaglio et al., 2018). Since its introduction, nudging has been studied and applied extensively in a great number of disciplines, like public administration (Bhanot \& Linos, 2020; Linos et al., 2020), but also including nutrition sciences (e.g., Bucher et al., 2016), environmental policy (e.g., Ölander \& Thøgersen, 2014), and health policy sciences (e.g., Marteau et al., 2011).

In the wake of its increasing popularity, nudging has attracted criticism, especially regarding its underlying ideology of what Thaler and Sunstein (2008) call 'libertarian paternalism'. The main premise of this critique is that nudges - while prevailing the option to choose - are still paternalistic and reduce the autonomy of individuals (Hausman \& Welch, 2010). However, others argue that restricting individual autonomy is not inviolable (Conly, 2012), and in this sense, a 'minor' autonomy violation caused by a nudge might not be that problematic. Moreover, some argue that nudges do not even constitute a violation of autonomy, as they help individuals to be autonomous in making the choices they actually want (De Ridder et al., 2020).

A second point of criticism relates to the conditions under which nudging is effective. A nudge may not work the way intended by the choice architect. Sunstein (2017) provides several reasons for why nudges might be ineffective. First, people may have strong preferences, and these preferences are hard to override with a nudge. Second, nudges can cause reactance among those being nudged or inadvertently cause compensating behavior. Finally, nudges must compete with counter-nudges. In other words, a nudge needs to stand out from other nudges, but this is often not the case (Cronqvist et al., 2018).

A third point of debate focuses on the duration of nudge effects. Allcott and Rogers (2014) found that the initial effects of a nudge-type intervention, in a report on energy use, wore off gradually when the intervention was abandoned. That being said, people kept responding to the intervention even though they were exposed to it for 2 years. In another study, Frey and Rogers (2014) argue that persistent behavioral change requires not only a single intervention in the choice architecture, but also building psychological habits, external reification, and changing what and how people think. Overall, nudges can be effective and persistent but only under a set of rather stringent conditions.

Hertwig and Grüne-Yanoff (2017) propose that in cases where nudges may be less effective, a 'boost' intervention can be considered. In contrast to adapting the decision environment to the confined mental state of an individual, boosting aims to enhance 
Table 1. Characteristics of nudging and boosting.

\begin{tabular}{lll}
\hline & Nudging & Boosting \\
\hline Perception of environment & Intervention target & Additional factor \\
\hline $\begin{array}{l}\text { Effect of environment on individual } \\
\text { repertoire }\end{array}$ & Triggering & Informing \\
\hline Perception of individual repertoire & $\begin{array}{c}\text { Almost nonmalleable } \\
\text { factor }\end{array}$ & $\begin{array}{c}\text { Malleable intervention } \\
\text { target }\end{array}$ \\
\hline Main success factor & Trigger stability & $\begin{array}{c}\text { Motivation and } \\
\text { teachability }\end{array}$ \\
\hline Main decision-making process & Reflexive & Reflective \\
\hline
\end{tabular}

Adapted from Grüne-Yanoff et al. (2018) and Hertwig and Grüne-Yanoff (2017).

the decision-making of an individual. Specifically, its goal is to improve knowledge or competences.

Although boosting, like nudging, acknowledges the bounded rationality of human decision-making, it draws different conclusions from it. Instead of changing the choice architecture, boosting approaches attempt to change skills, knowledge, or decision tools $(2017$, p. 974) in order to solve the misfit between the decision-maker and their environment (Hertwig, 2017, p. 146). For some, a boost may resemble the quite classic concepts of training or educational interventions. However, Hertwig and Grüne-Yanoff argue that boosting differs in a number of aspects, among which boosts should be informed by behavioral science evidence and boosts 'preserve and enable individuals' personal agency and autonomy' (2017, p. 982).

Theoretically, the core distinctions between nudging and boosting are their different foci (Table 1) (Hertwig, 2017; Grüne-Yanoff et al., 2018; Lorenz-Spreen et al., 2020). A nudge focuses on the decision environment, which is argued to trigger individuals into a certain behavior. Meanwhile, the individual repertoire is seen as a nearly nonmalleable factor. The focus of a boost is almost the opposite. The individual repertoire is the focus of the intervention, and as long as individuals are teachable and motivated, boosting can be successful in extending that repertoire. The decision environment is only an additional factor. Because of these different approaches, nudging and boosting are said to lead to different decision-making processes. A nudge leads to a reflexive decision-making process (a person reacts in a reflex on a change in the environment), and a boost leads to a reflective decision-making process (the reaction of a person is based on a change in knowledge and reflection hereon).

It should be noted that these differences are ideal types. In practice, many nudges tend to have some elements that can be classified as typical for boosts and vice versa. For instance, a 'simplify the message' nudge may increase an individual's knowledge on a certain subject. At the same time, a boost that conveys risk information may be argued to resemble an educational nudge. Hertwig (2017) acknowledges that the part where boosts provide knowledge is identical to an educational nudge, but that boosts stand out because, besides providing information, they attempt to improve decisionmaking competences, by, for example, using frequencies rather than probabilities. Hertwig (2017, p. 146) further argues: 'Rather than merely presenting pertinent 
and accurate information (as educative nudges do), boosts explicitly seek to foster existing decision-making competences and to develop new ones, thus enabling individuals to translate their intentions (preferences) into behaviour - that is, to exercise personal agency.' In sum, while nudges and boosts may show resemblance in parts, the whole of the intervention is distinctive.

Altogether the difference between nudging and boosting is open to debate, and theoretical distinctions may be harder to uphold in practical interventions (Hertwig, 2017). In our interventions, we opted for a pragmatic approach: we designed interventions that are theoretically fueled by either nudging or boosting, yet we acknowledge that one can find some level of congruence between the two, partly depending on one's vision of what nudging is, and consequently, what boosting adds. We will discuss this in more detail in the 'Methods' section.

\section{Potential effects of nudges and boosts on nurses}

So far, we have discussed the concepts of nudging and boosting from a generic theoretical perspective. In this section, we hypothesize how public professionals are affected by these techniques. Such a theoretical exploration is important, because public professionals such as nurses work in a specific organizational context, which is relevant for the effectiveness of the interventions. It is generally acknowledged that the work of public professionals has been changing in the past two decades. Public professionals in healthcare, particularly within hospitals, are not only expected to 'just' help patients, but also to work efficiently and to take accountability for their actions (Leicht et al., 2009). This has made the work of these public professionals much more complex and demanding.

In healthcare, nurses face a particularly demanding work environment. They usually work with multiple patients in multidisciplinary teams in which they have to constantly balance different priorities (Noordegraaf, 2016). At the same time, nurses have had to deal with societal and organizational demands for stricter surveillance and heightened accountability (Noordegraaf, 2007). Such demands may be translated into top-down plans and policies, which, especially when they are not in line with professional values, are likely to alienate public professionals from their profession (Tummers et al., 2009).

Understandably, in such a complex and changing work environment, it is hard to comply with 'yet another' protocol. Research has shown that protocol compliance does not depend on a rigid enforcement of the protocol by, for instance, punishing noncompliance, but rather on some way of gentle persuasion that the protocol is useful to the professional (Mikkelsen et al., 2017). For instance, Weske et al. (2019) find that medical doctors were more likely to comply with protocols when they had intrinsic commitment to comply. It is especially in these cases that behavioral interventions can be useful: hand hygiene protocols serve an important purpose, but a rigid enforcement approach is unlikely to be effective. Instead, behavioral interventions such as nudging and boosting can help persuade public professionals to comply.

Based on the literature, a nudge is likely to positively affect hand hygiene compliance. A recent systematic review by Nagtegaal et al. (2019) summarizes the literature on nudging healthcare professionals. They found 101 studies, of which $18 \%$ 
attempted to improve hand hygiene compliance. Of the hand hygiene nudges described in the studies, the large majority (77\%) was successful. This made hand hygiene compliance the most effective targeted outcome in the review.

However, the literature review by Nagtegaal et al. (2019) also shows that often similar nudge interventions were tested, and others have been ignored. For instance, most hand hygiene nudges were found to be mostly altering option-related efforts (e.g., changing dispenser locations). We further develop this stream of literature by testing a nudge that was used less but still easy to apply in a hospital setting. In particular, information reframing is a suitable nudge in this context, as this type of nudge has proven effective in a range of other settings (e.g., Neudecker et al., 2014; Mulderrig, 2017). However, it has not received much attention in the healthcare domain. Reframing is defined as 'shifting the perspectives of decision makers in ways that change their subjective evaluations of choice options' (Weber, 2012, p. 387). An example of a reframing nudge is to reframe the issue of blood donations as preventing deaths instead of saving lives, which increased donations (Chou \& Murnighan, 2013).

One study applied a reframing nudge in the hand hygiene context: Grant and Hofmann (2011) found increases in hand hygiene when professionals were reminded of the implications for patients, instead of how hand hygiene affects themselves. Here, we build on this study and see if reframing hand hygiene as a moment of patient care increases nurses' hand hygiene compliance. By reframing hand hygiene compliance as a way to show care for a patient, we allude to nurses' public service motivation. It has been shown that nurses perform better when they have a positive impact on the lives of others, such as patients (Bellé, 2013, 2014). This leads to the first hypothesis. ${ }^{1}$

H1: A nudging intervention will significantly increase the level of hand hygiene compliance, compared to a control group and pretests.

For boosting, no studies were found that explicitly use the approach of boosting in the context of hand hygiene. Yet, sometimes hand hygiene compliance interventions resembled a boost. For instance, a number of studies with effective interventions on hand hygiene included a form of on-the-job education (e.g., Bischoff et al., 2000). Educational interventions resemble boosts in the sense that they address individual learning but, as already explained, are not necessarily the same (Hertwig \& Grüne-Yanoff, 2017).

One important way in which relatively simple boosts can be delivered is through increasing people's risk literacy (e.g., Hoffrage et al., 2000; Gigerenzer et al., 2007). This approach suggests that people make wrong decisions because they misunderstand information about the associated risks. Hence, increasing their understanding of risks, their so-called risk literacy, can improve hand hygiene compliance. Ways to improve risk literacy make use of graphical representations (e.g., Lusardi et al., 2014), which eliminates biasing framing effects by using frequencies rather than probabilities because people tend to be biased in their risk estimation when confronted

\footnotetext{
${ }^{1}$ The hypotheses have been slightly reformulated from the preregistration without losing their original meaning.
} 
with probabilities (e.g., Gigerenzer et al., 2007), or training math skills (e.g., Berkowitz et al., 2015).

Increasing risk literacy is likely to improve hand hygiene compliance. Studies have shown that underestimating the importance of hand hygiene compliance and risks of noncompliance leads to worse hand hygiene (e.g., Dyson et al., 2011). At the same time, increasing risk literacy has been argued to be of vital importance for healthcare in general. For instance, Anderson et al. (2014) argue that efforts should be made to increase physician statistical literacy, as not doing this may lead to inaccurate risk estimates and misinformed patients.

In sum, we expect boosting to be an effective approach in increasing hand hygiene compliance.

H2: A boosting intervention will significantly increase the level of hand hygiene compliance, compared to a control group and pretests.

Nudging is a useful technique to help individuals make decisions that they wish to make when they have limited cognitive resources available (Hertwig, 2017, pp. 150-1). As their reaction is reflexive, its effects will be quickly visible. In contrast, the results of a boost may not immediately be visible, as it may take individuals longer to reflect, that is, for the new knowledge or skills to be developed (cf., e.g., Berkowitz et al., 2015). What is more, a person needs to possess some cognitive ability, resources, and motivation for the boost to be effective in the first place. Previous research shows that potential basic abilities include numerical or arithmetic skills (Sedlmeier \& Gigerenzer, 2001; Ramirez \& Beilock, 2011).

Another important argument in the debate on the effectiveness of boosts is that boosting is expected to have a more persistent effect than nudges: altering individuals' capacities through learning is supposed to lead to new heuristics that are remembered and play an active role in individuals' decision-making, whereas there are multiple ways in which nudges might fade (Hertwig, 2017). For instance, individuals may get used to changes in the environment or counter-nudges to mitigate the desired effect of a nudge (Sunstein, 2017; Cronqvist et al., 2018).

In sum, we expect nudging to have a larger immediate effect than boosting. Furthermore, we expect that public professionals, such as nurses, do possess skills and motivation to understand and apply hand hygiene protocols. Once they received a boost intervention and have learned about the importance and risks of (non)compliance, we expect this competence to be part of their professional expertise and thus to last until after the intervention has been removed, leaving a more persistent effect than the nudge intervention.

Hence, our last two hypotheses describe the expected difference between the interventions in their direct effect and their effect over time.

H3: Directly after the intervention, the nudging intervention will have a larger effect on the level of hand hygiene compliance than the boosting intervention.

H4: After the intervention is removed, the boosting intervention will have a larger effect on the level of hand hygiene compliance than the nudging intervention. 
Table 2. Experimental wards and attributed treatments.

\begin{tabular}{llcc}
\hline & Ward 1 & Ward 2 & Ward 3 \\
\hline $\begin{array}{c}\text { Discipline } \\
\text { MDL ('Maag-Darm- } \\
\text { Leverziekten': stomach, } \\
\text { intestines, and liver care) }\end{array}$ & $\begin{array}{c}\text { Day treatment } \\
\text { (brief } \\
\text { procedures) }\end{array}$ & $\begin{array}{c}\text { Orthopedics } \\
\text { (support or } \\
\text { movement } \\
\text { care) }\end{array}$ \\
\hline $\begin{array}{c}\text { Number of } \\
\text { beds }\end{array}$ & 17 & $39^{\text {a }}$ & 18 \\
\hline $\begin{array}{c}\text { Placement of } \\
\text { dispensers }\end{array}$ & Alcohol dispensers are placed at the entrance, near sinks, at beds, and in hallways. \\
\hline Treatment & Soap dispensers are placed near sinks. & Control group \\
\hline
\end{tabular}

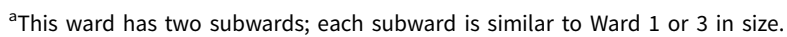

\section{Methods}

\section{Preregistration and publication of data}

The research question and hypotheses were submitted to an online registry, Evidence in Governance and Politics [EGAP, now accessible through the Open Science Framework (OSF)], prior to the execution of the experiment. The registration number is 20190313AA (Van Roekel, 2020). For research transparency reasons and as an addition to the information in this article, a number of appendices were uploaded to the OSF and can be viewed at this link: https://osf.io/tyvdx/?view_only=53dle2a43c6a40e79776566bcdcbaed5. The appendices present an anonymous copy of the preregistration (Supplementary Appendix 1), experimental visuals (Supplementary Appendix 2), the structured observation scoring sheet (Supplementary Appendix 3), the data (Supplementary Appendix 4), and additional logistic regression analyses (Supplementary Appendix 5).

\section{Setting and participants}

The experiment took place in a regional hospital in a large Dutch city in April 2019. The hospital has over 300 beds and roughly 2000 employees, medical specialists, and volunteers and delivers healthcare in a variety of specialisms and wards. Within the hospital, three wards participated in the experiment and formed three experimental groups. The ward characteristics are presented in Table 2. While each of the three wards was randomly allocated to either one of the treatments or no treatment, the total of three wards to participate in the experiment was chosen purposefully, due to the low number of wards in the hospital. Furthermore, randomization of treatments within wards was not employed, but it would likely cause treatment diffusion: nurses within wards are in direct contact and could exchange information. In contrast, treatment diffusion across wards is unlikely. In interviews with ward supervisors and nurses (see 'prestudy'), nurses stated to rarely visit other wards, as every ward focuses on a different medical discipline with its own pool of specialized nurses.

Quasi-experimentation is a valuable method in assessing causality when fully randomized designs are not possible or desirable (Shadish et al., 2002; Grant \& Wall, 2009). 


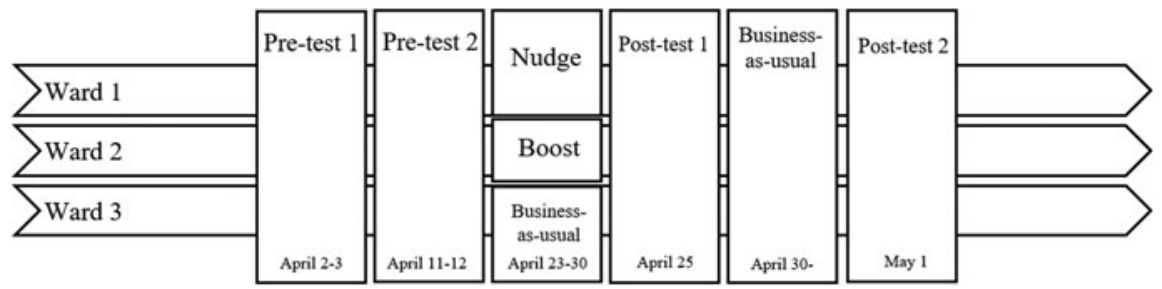

Figure 1. Experimental procedure (all dates refer to 2019).

To further increase internal validity, the three wards were selected based on their similarities: all wards provide nonintensive care to a large variety of patients and have similar ward and personnel structures. Interviews and observations confirmed that similar numbers of nurses are at work in the several (sub)wards, as similar numbers of beds are located there (17-20 beds; Ward 2 has 39 beds in total, but these are distributed over two separate subwards so that nurses work in a very similar environment compared with Ward 1 or 3). Finally, peak hours overlap: ward heads confirm that peak hours usually occur between 9 AM and 3 PM. However, the exact numbers of patients could vary per day and per ward, and this is something that could not be accounted for.

\section{Design and procedure}

This study adopted a quasi-experimental design and included a control group and pre- and post-tests. Double pretests were conducted to reduce threats to internal validity due to potential inherent differences between the groups (Shadish et al., 2002, p. 145), and double post-tests were used to assess the longitudinal intervention effect after intervention removal.

Before the experiment, a qualitative prestudy was conducted to develop the interventions. This prestudy took place from December 2018 until March 2019. Next, the experiment started (Figure 1 outlines the procedure). Two pretests were conducted before the interventions were implemented. At the same time, the control group received no treatment (business as usual). A post-test followed, after which the interventions were removed and another post-test followed. After intervention removal, all wards returned to business as usual.

\section{Prestudy}

We followed a recommended procedure by Münscher et al. (2016) to develop our behavioral interventions. A qualitative prestudy was conducted to find out what hindrances employees of the hospital experienced in complying with the hand hygiene protocol and what specific types of nudging and boosting seemed promising to improve compliance. Data were collected through 11 semistructured interviews with ward heads and nurses, and document analysis. We followed a four-step model to (1) define the behavioral problem, (2) analyze the applicability of behavioral interventions, (3) check for behavioral bottlenecks, and (4) build hypotheses on behavioral interventions. 
The behavioral problem at stake was low compliance levels of nurses with the hand hygiene protocol. The choice architecture was arguably a good fit for this study, as in the interviews, behavioral factors were dominant, and other factors, like deliberate opposition toward protocols, were never sole forces of noncompliance. Two main factors arose within all three wards that especially limited hand hygiene compliance. First, nurses had negative perceptions of the hand hygiene protocol: it was perceived to be long, complicated, hard to remember, and burdensome. Second, nurses wondered why they needed to comply so strictly and to what extent noncompliance would be problematic: questions were asked about the specifications, the relevance, and the urgency of the protocol. Finally, a specific type of nudging and boosting could be developed to address these two factors.

\section{Intervention development}

\section{Treatment aim and materials}

First, the nudging intervention (Ward 1) addressed the negative perceptions nurses had of the hand hygiene protocol through intervening in the decision environment of individuals. It tried to do so by reframing the message (Grant \& Hofmann, 2011; Münscher et al., 2016). Following the reframing-logic, it did so by reframing the hand hygiene protocol from an extra burden (a negative frame) to a moment of care for the patient (a positive frame). The tagline used for this intervention was 'in good hands', which, accompanied by an image of hands that are being cleaned, implicitly related caring for patients to having clean hands.

The boosting intervention (Ward 2) aimed to increase the understanding of risks involved in not following the hand hygiene protocol. As explained in the theoretical part of this article, boosting targets the individual repertoire by providing new heuristics. In this study, we used a risk literacy boost, as reasoning about risks is trainable with relative ease (Nisbett et al., 1987). Furthermore, work by Gigerenzer and colleagues (e.g., Sedlmeier \& Gigerenzer, 2001) has shown that risk literacy and statistical literacy can be greatly increased by presenting risks in a format using frequencies (i.e., absolute numbers) rather than probabilities (i.e., relative numbers). For instance, using a frequency format helps women to better estimate the risks associated with breast cancer screening (Gigerenzer, 2014).

In our study, the boosting intervention aimed to improve the nurses' understanding of the frequency of hospital infections and the role of hand hygiene of nurses in causing these infections. Although there is a broad understanding of the risks of lacking hand hygiene in the nurse profession, this risk is not always well understood (De Wandel et al., 2010). By providing information in a frequency format, the actual magnitude of the risk becomes clearer and helps them to better understand the risk of lacking hand hygiene.

We designed a poster with the tagline 'prevent infections'. On this poster, multiple facts about infection risks were listed in frequencies rather than probabilities. The key messages that nurses were confronted with were as follows (Pittet et al., 2006; Carboneau et al., 2010; IGJ, 2018): 
- 'One in every twenty patients receive a hospital-induced infection.'

- 'Research shows that in two American hospitals, the number of cases with MRSA (Methicillin-resistant Staphylococcus aureus) infections decreased by half after healthcare employees improved their hand hygiene.'

Finally, the control group (Ward 3) received no intervention and was subject to business as usual. In a business-as-usual situation, no special attention to the hand hygiene protocol was provided, but nurses had the possibility to read the protocol at a physical location in the ward as well as online. Hence, nurses in the control condition had equal opportunity to comply with the hand hygiene protocol.

\section{Treatment delivery}

Separate nudging and boosting interventions were set up in the wards. The content of the interventions was communicated via a poster and a flyer. First, the posters were placed at 10 highly visible locations in the wards. Second, flyers were placed on the table in the break room of each ward. Supplementary Appendix 2 presents a complete overview of the visuals. Third, supervisors notified their employees about the campaign by sending them an email that contained the flyer. The email was dictated by the researchers and read: Hello everyone, in the following weeks we will give extra attention to the hand hygiene protocol. Posters will be hung in the wards and flyers will be distributed. Please view the appendix, there you will find the flyer too. Good luck!

From a methodological point of view, this email could be considered a treatment in itself. While we agree with this point, this should be seen as a 'lesser evil' than the alternative of not announcing the poster campaign. It would occur very strange to the ward employees if suddenly there were posters in the wards that were not announced, which would harm the external validity of our study.

In this field setting, the support of ward supervisors is crucial: they have to agree to changes made in their wards to prevent resistance or the chance of having to take the posters down. The important role of ward supervisors may give cause to worry about idiosyncrasies regarding treatment delivery or treatment effects, dependent on supervisor characteristics. However, there is little reason to assume that variation across ward supervisors had a sizable impact, as all supervisors had a similar leadership style: they were highly involved with their ward, and nurses and ward cultures are nonhierarchical (e.g., our prestudy indicates that in all wards, nurses came into the head office to casually talk to their ward heads).

\section{Treatment differences and similarities}

From a theoretical point of view, the crucial difference between nudges and boosts is the element they target in the decision-making process. Nudges target the decision frame, whereas boosts seek to provide agents with additional heuristics in the decision-making process. In field settings, it can be harder to distinguish the two approaches. In such a setting, interventions need to be suitable and acceptable in that particular (hospital) context.

For instance, the nudge message also contained information about the five 'hand hygiene moments', which is providing more information than merely reframing. The 
boost intervention contained nudge-like elements, as it is presented through posters and relatively simple messages. In doing so, it makes decision information salient, which is also considered a nudge technique (Münscher et al., 2016). Still, the boost intervention is predominantly 'boosting'. The fact that noncompliance increases infection risks is known to nurses, and in that sense, the information presented is not new. Therefore, presenting infection risks using a risk literacy intervention (e.g., Gigerenzer et al., 2007) was aimed at helping nurses to better understand the risks of bad hand hygiene.

Altogether, we acknowledge that the nudge versus boost distinction in the literature is ideal-typical, and that in practice, some elements of boosts and nudges can be seen in both approaches. Nevertheless, there are important differences, and both interventions clearly have a different emphasis in line with the theoretical distinction between nudges and boosts.

\section{Measurement}

Hand hygiene compliance was measured through standardized observations. Importantly, the units of analysis are not nurses but the number of potential hand hygiene moments in a ward for nurses. We used a previously tested and validated approach and protocol for our observations (Supplementary Appendix 3; cf., e.g., Sax et al., 2009; Erasmus et al., 2010; King et al., 2016). There were four observation rounds: two before the intervention, one right after the intervention was implemented, and one after the intervention was removed from the wards. For each observation round, each of the wards was observed for approximately $2 \mathrm{~h}$ between $9 \mathrm{AM}$ and 3 PM, within 2 days, during what ward heads defined as peak hours. During this time, nurses at work in the ward at that moment were individually observed, as many as possible and one by one, to avoid a situation where one specific nurse would skew the results. An individual nurse was selected through random convenience sampling (meaning the nurse had to be working with patients at that very moment; Sax et al., 2009) and continuously observed until they seemed to have finished patient contact (by, e.g., leaving the ward or moving on to computer work) or, in the rare case this did not happen, for a maximum of $15 \mathrm{~min}$.

During the close observation, every potential moment of hand hygiene was noted (cf., WHO, 2009). After noting the potential moment, the reaction of the nurses was noted: Was hand hygiene applied by means of alcohol or water and soap, or not applied? Both forms of cleaning were noted as compliance. For more details on this approach of hand hygiene observations, as developed by the WHO, see Sax et al. (2009). To be able to conduct these observations correctly, the first author attended a hand hygiene observation training at another hospital. This training was given by infection prevention experts and nurses.

\section{Analysis}

A quantitative analysis of the observational data was conducted. The statistical significance of the observations was assessed using generalized estimating equations (GEEs). GEEs provide, in short, a semiparametric way to analyze longitudinally correlated data (Hanley et al., 2003). Our model assumes independence of observations between 


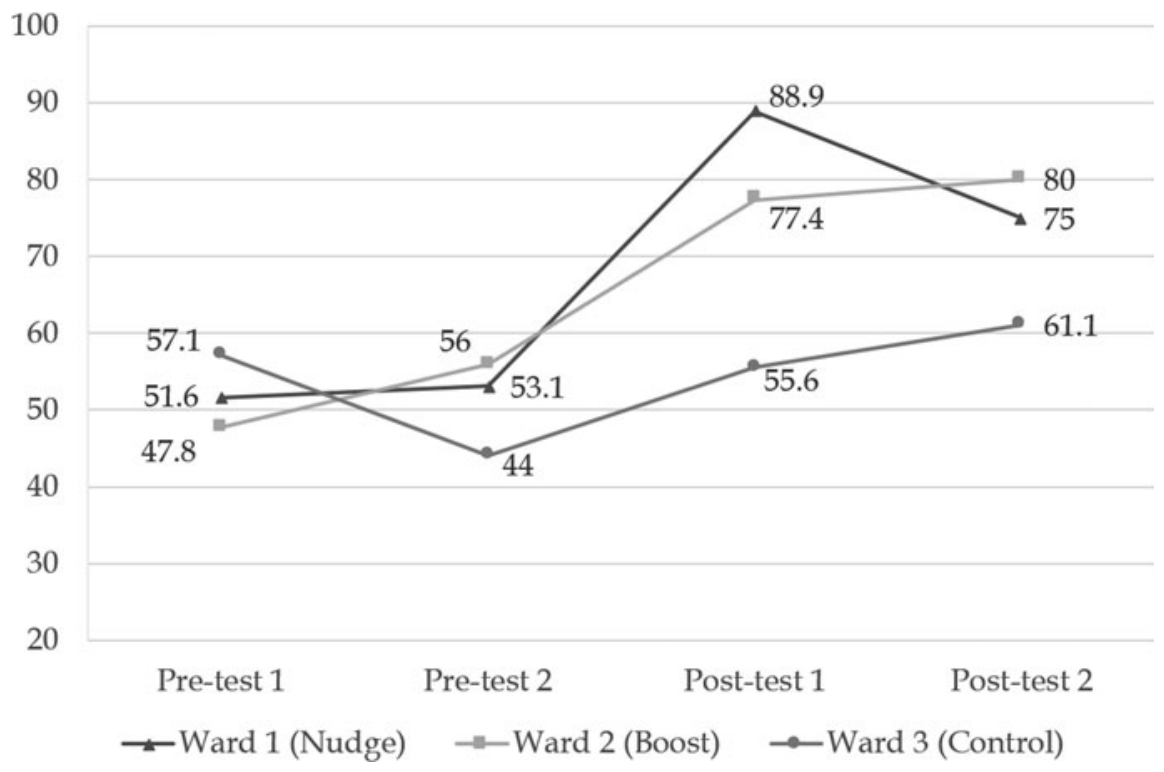

Figure 2. Compliance scores for hand hygiene in percent. The total number of observations is 348 ( 98 , 107, 76, and 67 for pretests 1 and 2 and post-tests 1 and 2, respectively.

subjects (in our case: wards). However, in contrast to logistic regression analysis, it allows for the data to be dependent within subjects. GEEs are suitable for a binary dependent variable (in this case, compliance) and categorical independent variables (in this case, the wards with Ward 3 as reference, and the pre- and post-tests with the first pretest as reference) (Hanley et al., 2003). Using a robust estimator, we employ a Type-3 analysis to test model effects and compute Wald Chi-Square statistics, with $95 \%$ confidence intervals. The link function is logit for a binomial distribution: the dependent variable is binary (compliance or noncompliance). Within-subject correlation is assumed independent. We calculate both main effects for all wards and tests and their interaction effects.

Because dependence within wards is likely for a repeated-measure design like this one (measuring compliance over time), this makes logistic regression a less fitting candidate for the analysis. In these circumstances, GEEs are found to provide closer approximations of population averages (Hubbard et al., 2010). However, Supplementary Appendix 5 presents logistic regression analyses to compare the GEE and provide explained variance estimates. These results should be viewed with more caution for reasons explained above, but as can be seen, there are no substantial differences between the results of the two analyses.

\section{Results}

\section{Observations}

In total, 348 moments of potential hand hygiene compliance were observed. In Figure 2, the compliance scores (i.e., the ratio of moments of actual compliance 
Table 3. Coefficients, standard errors, p-values, and confidence intervals of GEEs.

\begin{tabular}{lccc}
\hline Dependent variable: compliance & $B(\mathrm{SE})$ & $\mathrm{p}$ & $95 \% \mathrm{Cl}$ \\
\hline Ward 1 (nudge) & $-0.22(0.57)$ & 0.70 & $(-1.34,0.89)$ \\
\hline Ward 2 (boost) & $-0.38(0.53)$ & 0.48 & $(-1.42,0.67)$ \\
\hline Pretest 2 & $-0.53(0.60)$ & 0.38 & $(-1.70,0.64)$ \\
\hline Post-test 1 & $-0.07(0.59)$ & 0.91 & $(-1.22,1.09)$ \\
\hline Post-test 2 & $0.16(0.65)$ & 0.80 & $(-1.12,1.45)$ \\
\hline Ward 1 × Pretest 2 & $0.59(0.78)$ & 0.45 & $(-0.94,2.12)$ \\
\hline Ward 1 × Post-test 1 & $2.08^{\star *}(1.02)$ & 0.04 & $(0.08,4.07)$ \\
\hline Ward 1 $\times$ Post-test 2 & $0.87(0.88)$ & 0.33 & $(-0.86,2.60)$ \\
\hline Ward 2 $\times$ Pretest 2 & $0.86(0.72)$ & 0.24 & $(-0.56,2.27)$ \\
\hline Ward 2 $\times$ Post-test 1 & $1.38^{\star}(0.78)$ & 0.08 & $(-0.16,2.92)$ \\
\hline Ward 2 $\times$ Post-test 2 & $1.31(0.87)$ & 0.14 & $(-0.41,3.02)$ \\
\hline Constant & $0.29(0.44)$ & 0.51 & $(-0.58,1.15)$ \\
\hline$N$ & 348 & & \\
\hline
\end{tabular}

Pretest 1 and Ward 3 (control) are taken as reference categories. ${ }^{*} p<0.1,{ }^{\star *} p<0.05$.

and all potential moments) per ward and per round are presented. For example, when four potential moments were observed and in two of them the nurse applied hand hygiene, it led to a score of 50\%. Notably, Wards 1 and 2 showed more or less comparable scores for the two pretests. Next, they received an intervention and subsequently showed a large increase in compliance in post-test 1 with $+21.4 \%$ and $+35.8 \%$, respectively. Particularly, the nudge intervention (Ward 1) had a strong increase in compliance. In post-test 2, a week later and after intervention removal, Ward 2 (subject to the boost) increased slightly (+2.6\%), while Ward 1 showed a decrease $(-13.9 \%)$. Meanwhile, the control group showed a decrease in pretest 2 and gradual increases in subsequent rounds, yet these were minor compared with the intervention groups.

Table 3 presents a full model with main effects and interactions for all wards and tests. The interactions for the nudge and the boost with the first post-test are significant at $p<0.05$ and $p<0.1$, respectively. This supports the idea that both interventions positively increased hand hygiene compliance. We do not see significant effects in the second post-test, which seems odd because the mean compliance score for Ward 2 (boost) does slightly increase between post-test 1 and post-test 2. One explanation is that there were slightly less observations in the second post-test, leaving us with less statistical power to detect effects. Also, compliance in the control increased, albeit insignificantly.

To further probe our hypotheses, we carried out three separate GEEs: one for each of the wards. This decreases the number of factors in the model because we only take time into account and remove interactions as well as the analysis of multiple wards within one model. This has the disadvantage of not being able to compare the results 
of the intervention vis-à-vis the control group but has the advantage of more statistical power while still being able to compare across time. This analysis is complementary to the one in Table 3.

The analyses per ward (Table 4 ) show significant results for all post-tests in the ward subject to the boost $(p<0.05)$. The highest coefficient is found for the second post-test. For the ward subject to the nudge, the first post-test is significant at $p<0.05$, while the second post-test is significant at $p<0.1$ with a notably smaller coefficient. There are no significant differences between the pretests for all wards, nor for any of the tests in the control group. Explained variance scores are not provided for GEEs, but Supplementary Appendix 5 presents logistic regression analyses that present almost identical results and that do provide Nagelkerke's $R^{2}$. For the full model, $R^{2}$ is 0.10 . For the models per ward, the $R^{2}$ scores are $0.13,0.10$, and 0.02 for Wards 1,2 , and 3 , respectively.

\section{Hypotheses}

In sum, Table 5 shows the results of our hypotheses. First, Hypotheses 1 and 2 receive the clearest support. Both interventions significantly affected nurses' compliance with the hand hygiene protocol. Further support of their effectiveness was presented by the control group, where no significant changes had been observed.

Next, Hypothesis 3 predicted that the nudge would have a stronger effect than the boost right after the intervention. We find that there is some support for this. Although the overall differences are small, we find that the nudge in post-test 1 has a higher coefficient than the boost in post-test 1 . Hypothesis 4 postulated that after the interventions were removed (post-test 2), the boosting intervention would have a larger effect than the nudge. We do not find these effects in the main model, but we do find support for this hypothesis in our additional analysis where the three wards are analyzed separately. In additional analysis, the coefficient in the boost condition increases, whereas the coefficient decreases in the nudge group. In sum, although there seems to be some preliminary support for Hypothesis 4, we should interpret these results with caution.

\section{Discussion}

During a 4-week nonrandomized quasi-experiment, we tested and compared a nudge and boost intervention in a highly relevant field setting: hand hygiene compliance by nurses in a hospital. Based on our findings, we answer the following central question: What is the effect of nudging and boosting on hand hygiene compliance of nurses?

The results showed that both a nudging intervention that aims to reframe the protocol from a patient perspective and a boosting intervention that aims to increase the risk literacy of nurses through providing information about infections led to significantly higher compliance than the preintervention levels. The control group did not show significant differences during the intervention period. This means that both types of interventions were effective in increasing hand hygiene among nurses.

Furthermore, the results suggest that there might be a difference in effectiveness between the nudge and boost after intervention removal. When we removed the interventions, the ward exposed to the boosting intervention remained on a similarly high level of compliance, while this effect faded somewhat in the ward that was exposed to 
Table 4. Coefficients, standard errors, $p$-values, and confidence intervals of GEEs for all wards.

\begin{tabular}{|c|c|c|c|c|c|c|c|c|c|}
\hline \multicolumn{10}{|c|}{ Dependent variable: compliance } \\
\hline Pretest 2 & $0.06(0.50)$ & 0.90 & $(-0.93,1.05)$ & $0.33(0.41)$ & 0.42 & $(-0.48,1.13)$ & $-0.53(0.60)$ & 0.38 & $(-1.70,0.64)$ \\
\hline Post-test 1 & $2.02^{\star \star}(0.83)$ & 0.02 & $(0.39,3.65)$ & $1.32^{\star \star}(0.52)$ & 0.01 & $(0.30,2.34)$ & $-0.07(0.59)$ & 0.91 & $(-1.22,1.09)$ \\
\hline$N$ & 105 & & & 152 & & & 91 & & \\
\hline
\end{tabular}

Pretest 1 is taken as a reference category.

${ }^{\star} p<0.1,{ }^{\star *} p<0.05$. 
Table 5. Hypotheses.

\begin{tabular}{ll}
\hline Hypothesis & Finding \\
\hline $\begin{array}{l}\text { H1: A nudging intervention will significantly } \\
\text { increase the level of hand hygiene } \\
\text { compliance }\end{array}$ & Supported \\
\hline $\begin{array}{l}\text { H2: A boosting intervention will significantly } \\
\text { increase the level of hand hygiene } \\
\text { compliance }\end{array}$ & $\begin{array}{l}\text { Supported } \\
\text { H3: Directly after the intervention, the nudging } \\
\text { intervention will have a larger effect on the } \\
\text { level of hand hygiene compliance than the } \\
\text { boosting intervention }\end{array}$ \\
$\begin{array}{l}\text { H4: After the intervention is removed, the } \\
\text { boosting intervention will have a larger } \\
\text { effect on the level of hand hygiene } \\
\text { compliance than the nudging intervention }\end{array}$ & $\begin{array}{c}\text { Supported. After the intervention, the effect } \\
\text { for nudging is stronger than that for } \\
\text { boosting }\end{array}$ \\
\hline
\end{tabular}

the reframe nudge. Although we acknowledge that we would need additional observations to see if this trend indeed follows through, it is an intriguing finding which if confirmed - would be in line with theoretical expectations (Grüne-Yanoff et al., 2018).

Yet, even without considering the potential difference in the duration of the effect, our finding is of interest. We show that boosting and nudging can both be effective in a complex hospital environment. This implies that boosting provides an alternative to behavioral change in a similarly cost-effective way as nudging. We provide evidence that even in a high-stakes, high-pressure environment such as hospitals, boosts can serve as an effective intervention in addition to nudges.

\section{Limitations}

Before discussing the implications of these results, we highlight some limitations to this study. First, although we carefully designed a boosting and nudging intervention based on their theoretical distinctions, in practice, the difference between a boost and a nudge appeared to be somewhat tenuous. For instance, some elements of nudge can be identified in the boost intervention, which makes for a slightly weaker test of the theory. In our view, this also indicates that theoretical distinctions may not always be feasible or realistic outside of 'clean' lab or survey environments. In field settings, researchers deal with less control of the setting and intervention, which results in a 'messier' but more realistic test (Harrison \& List, 2004). Still, for the purposes of theory testing, future research may try to provide for a purer empirical test to compare the effectiveness of nudge versus boost through lab or survey experiments (e.g., Bradt, 2019).

A second issue is that we need to gain stronger evidence on the persistence of boosting interventions. Although the maintained timeline to observe effects is not unusual in these types of experiments (e.g., Erasmus et al., 2010; Grant \& Hofmann, 2011), we do not know if the effect of the boost lasted in the weeks after the experiment ended. Therefore, we recommend future studies to take longer 
timelines and more observations into consideration when testing a boosting or nudging intervention. This will also provide the power to detect longer-lasting effects. To increase power, we partially base the persistence of the boost on within-ward analyses without comparing between the wards. Without a control group, results may be biased through trends unrelated to the treatment, like events occurring during treatment, natural maturation of participants, or pretest effects (Shadish et al., 2002). Although our design took various measures to eliminate such threats to internal validity (e.g., double pre- and post-tests), future research may substantiate our tentative interpretation of the effect of the boost intervention by increasing the number of observations and length of the time frame and possibly look for ways to truly randomize the treatment.

Finally, observation and observer bias might be an issue since the compliance behavior of nurses was observed in an obtrusive manner and nurses may be influenced by the presence of the observer. Multiple measures were taken to minimize demand effects, such as observing the nurses for a longer period of time so that nurses' consciousness of the observer would diminish (Erasmus et al., 2010; Grant \& Hofmann, 2011) and adding a control group. Still, some observer bias may still have been present because we could not apply a double-blind procedure: the observant could see the intervention while observing hand hygiene behavior. Nevertheless, observant biases have been reduced by taking a validated hand hygiene training and by supervision by the second and third authors, as well as the infection prevention department in the hospital.

\section{Implications}

Our findings have important implications for theory. First, scientists and scholars in behavioral public administration have been debating the potential effects of nudging versus boosting mostly in theoretical terms (Hertwig, 2017; Hertwig \& Grüne-Yanoff, 2017; Grüne-Yanoff et al., 2018), yet they have provided limited empirical evidence comparing the two types of interventions so far. Interestingly, one explicit nudge/ boost comparison by Bradt (2019) found that a boost was less effective than a nudge. Hence, our finding that a boost is at least as effective as a nudge in a highly relevant context indicates that we need to take the context into account when comparing the effectiveness of nudges against boosts.

This finding raises new questions about the conditions under which either nudges or boosts are likely to be more effective. Indeed, one of the conditions that needs more systematic analysis is the field context. Here, we find that in a hospital context, boosts are at least as effective as nudges. One reason for this might be that in this context cognitive capacities and motivation to process a boost are relatively high, which are indeed considered necessary conditions for a successful boost (Hertwig, 2017). Future research could address this issue by comparing the effectiveness of nudges and boosts by systematically varying contextual conditions, where we would expect differences in cognitive capabilities and/or motivation, such as the target audience (e.g., professionals vs. laymen) or the type of policy domain (e.g., finance, healthcare, and sustainability).

Indeed, the theoretical debate on nudges versus boosts already shows that boosts may work only when there is sufficient cognitive capacity and motivation 
(Hertwig, 2017). We do not argue that boosts are always equally or more effective than nudges, yet in contexts of professionals and in hospitals or other healthcare organizations, these preconditions are often present. Experimenting with boosts to improve specific behaviors of public professionals may, therefore, indeed be a promising new research avenue for behavioral scientists and scholars working in behavioral public administration (Tummers, 2019).

This article also has a practical implication. Public professionals often work in high-stakes and high-pressure environments (e.g., Noordegraaf \& Steijn, 2014; Tummers et al., 2015). We show that a relatively cheap nudging or boosting intervention can help public professionals to comply with important protocol demands. This answers to calls from scholars who emphasize that we should study the choice environment to change not only citizen behavior, but also the behavior of public professionals (e.g., Dudley \& Xie, 2019).

\section{Conclusion}

In this article, we show that nudges and boosts are both effective in a highly relevant field setting. The interventions increased hand hygiene compliance by 35.8 and 21.4 percentage points in the week after the interventions took place. Furthermore, our results provide an initial indication that the effect of a boost has the potential to persist even after intervention removal, although we admit that follow-up research is needed to confirm this finding. Hence, nudging and boosting might be vital interventions in combatting hospital infections, which has - after we carried out the experiment in the Spring of 2019 - become increasingly relevant due to the COVID-19 pandemic. Finally, nudging and boosting can help ensure that professionals such as nurses are able to do what they are trained to do while adhering to hand hygiene standards.

Acknowledgments. The authors wish to thank Lars Tummers, Betteco Slieker, and Femke de Vries for their mentorship in developing this study, Albert van Wijk and Eelco de Graaf for their support, and Renate Stevens and Nienke Cabell for their contributions to the poster design. Earlier versions of this article were presented at the NIG Annual Work Conference 2019 (November 7 and 8) in Amsterdam and the online Panel on Behavioral and Experimental Public Management on April 27, 2020. We thank the participants for their helpful comments and suggestions.

Supplementary material. To view supplementary material for this article, please visit https://doi.org/10. 1017/bpp.2021.15.

\section{References}

Allcott, H. and T. Rogers (2014), 'The short-run and long-run effects of behavioral interventions: Experimental evidence from energy conservation', American Economic Review, 104(10): 1-37.

Andersen, S. C. and U. Hvidman (2020), 'Can reminders and incentives improve implementation within government? Evidence from a field experiment', Journal of Public Administration Research and Theory. doi:10.1093/jopart/muaa022.

Anderson, B. L., G. Gigerenzer, S. Parker and J. Schulkin (2014), 'Statistical literacy in obstetricians and gynecologists', Journal for Healthcare Quality, 36(1): 5-17.

Battaglio, P. R. Jr., P. Belardinelli, N. Bellé and P. Cantarelli (2018), 'Behavioral public administration ad fontes: A synthesis of research on bounded rationality, cognitive biases, and nudging in public organizations', Public Administration Review, 79(3): 304-20. 
Bellé, N. (2013), 'Experimental evidence on the relationship between public service motivation and job performance', Public Administration Review, 73(1): 143-53.

Bellé, N. (2014), 'Leading to make a difference: A field experiment on the performance effects of transformational leadership, perceived social impact, and public service motivation', Journal of Public Administration Research and Theory, 24(1): 109-36.

Bellé, N., P. Cantarelli and P. Belardinelli (2018), 'Prospect theory goes public: Experimental evidence on cognitive biases in public policy and management decisions', Public Administration Review, 78(6): 828-40.

Berkowitz, T., M. W. Schaeffer, E. A. Maloney, L. Peterson, C. Gregor, S. C. Levine and S. L. Beilock (2015), 'Math at home adds up to achievement in school', Science, 350(6257): 196-8.

Bhanot, S. P. and E. Linos (2020), 'Behavioral public administration: Past, present, and future', Public Administration Review, 80(1): 168-71.

Bischoff, W. E., T. M. Reynolds, C. N. Sessler, M. B. Edmond and R. P. Wenzel (2000), 'Handwashing compliance by health care workers: The impact of introducing an accessible, alcohol-based hand antiseptic', Archives of Internal Medicine, 160(7): 1017-21.

Bradt, J. (2019), 'Comparing the effects of behaviorally informed interventions on flood insurance demand: An experimental analysis of "boosts" and "nudges", Behavioral Public Policy, 1-31. doi:10.1017/ bpp.2019.31.

Bucher, T., C. Collins, M. E. Rollo, T. A. McCaffrey, N. De Vlieger, D. Van der Bend, H. Truby and F. J. Perez-Cueto (2016), 'Nudging consumers towards healthier choices: A systematic review of positional influences on food choice', British Journal of Nutrition, 115(12): 2252-63.

Carboneau, C., E. Benge, M. T. Jaco and M. Robinson (2010), 'A lean Six Sigma team increases hand hygiene compliance and reduces hospital-acquired MRSA infections by 51\%', Journal for Healthcare Quality, 32(4): 61-70.

Chou, E. Y. and J. K. Murnighan (2013), 'Life or death decisions: Framing the call for help', PLoS One, 8(3): $1-6$.

Conly, S. (2012), Against autonomy: Justifying coercive paternalism. Oxford: Oxford University Press.

Cronqvist, H., R. H. Thaler and F. Yu (2018), 'When nudges are forever: Inertia in the Swedish premium pension plan', AEA Papers and Proceedings, 108: 153-8. doi:10.1257/pandp.20181096.

De Ridder, D., J. Feitsma, M. Van den Hoven, F. Kroese, T. Schillemans, M. Verweij and E. De Vet (2020), 'Simple nudges that are not so easy', Behavioural Public Policy, 1-19. doi:10.1017/bpp.2020.36.

De Wandel, D., L. Maes, S. Labeau, C. Vereecken and S. Blot (2010), 'Behavioral determinants of hand hygiene compliance in intensive care units', American Journal of Critical Care, 19(3): 230-9.

Drexler, A., G. Fischer and A. Schoar (2014), 'Keeping it simple: Financial literacy and rules of thumb', American Economic Journal: Applied Economics, 6(2): 1-31.

Dudley, S. E. and Z. Xie (2019), 'Designing a choice architecture for regulators', Public Administration Review, 80(1): 151-6.

Dyson, J., R. Lawton, C. Jackson and F. Cheater (2011), 'Does the use of a theoretical approach tell us more about hand hygiene behavior? The barriers and levers to hand hygiene', Journal of Infection Prevention, 12(1): 17-24.

Erasmus, V., M. N. Kuperus, J. H. Richardus, M. C. Vos, A. Oenema and E. F. Van Beeck (2010), 'Improving hand hygiene behavior of nurses using action planning: A pilot study in the intensive care unit and surgical ward', Journal of Hospital Infection, 76(2): 161-4.

Frey, E. and T. Rogers (2014), 'Persistence: How treatment effects persist after interventions stop', Policy Insights from the Behavioral and Brain Sciences, 1(1): 172-9.

Gigerenzer, G. (2014), 'Breast cancer screening pamphlets mislead women', British Medical Journal, 348. doi:10.1136/bmj.g2636.

Gigerenzer, G., W. Gaissmaier, E. Kurz-Milcke, L. M. Schwartz and S. Woloshin (2007), 'Helping doctors and patients to make sense of health statistics', Psychological Science in the Public Interest, 8(2): 53-96.

Grant, A. M. and D. A. Hofmann (2011), 'It's not all about me: Motivating hand hygiene among health care professionals by focusing on patients', Psychological Science, 22(12): 1494-9.

Grant, A. M. and T. D. Wall (2009), 'The neglected science and art of quasi-experimentation: Why-to, when-to, and how-to advice for organizational researchers', Organizational Research Methods, 12(4): $653-86$.

Grimmelikhuijsen, S., S. Jilke, A. L. Olsen and L. Tummers (2017), 'Behavioral public administration: Combining insights from public administration and psychology', Public Administration Review, 77(1): 45-56. 
Grüne-Yanoff, T., C. Marchionni and M. A. Feufel (2018), 'Toward a framework for selecting behavioral policies: How to choose between boosts and nudges', Economics \& Philosophy, 34(2): 243-66.

Hanley, J. A., A. Negassa and J. E. Forrester (2003), 'Statistical analysis of correlated data using generalized estimating equations: An orientation', American Journal of Epidemiology, 157(4): 364-75.

Harrison, G. W. and J. A. List (2004), 'Field experiments', Journal of Economic literature, 42(4): 1009-55.

Hausman, D. M. and B. Welch (2010), 'Debate: To nudge or not to nudge', Journal of Political Philosophy, 18(1): 123-36.

Hertwig, R. (2017), 'When to consider boosting: Some rules for policy-makers', Behavioral Public Policy, 1(2): 143-61.

Hertwig, R. and T. Grüne-Yanoff (2017), 'Nudging and boosting: Steering or empowering good decisions', Perspectives on Psychological Science, 12(6): 973-86.

Hoffrage, U., S. Lindsey, R. Hertwig and G. Gigerenzer (2000), 'Communicating statistical information', Science, 290: 2261-62. doi:10.1126/science.290.5500.2261.

Hubbard, A. E., J. Ahern, N. L. Fleischer, M. Van der Laan, S. A. Lippman, N. Jewell, T. Bruckner and W. A. Satariano (2010), 'To GEE or not to GEE: Comparing population average and mixed models for estimating the associations between neighborhood risk factors and health', Epidemiology, 21: 467-74.

Inspectie Gezondheidszorg en Jeugd (IGJ) (2018), Infectiepreventie, een kwestie van gedrag en een lange adem. Den Haag: Ministerie van Volksgezondheid, Welzijn en Sport.

Jones, B. D. (2017), 'Behavioral rationality as a foundation for public policy studies', Cognitive Systems Research, 43: 63-75.

King, D., I. Vlaev, R. Everett-Thomas, M. Fitzpatrick, A. Darzi and D. J. Birnbach (2016), “Priming" hand hygiene compliance in clinical environments', Health Psychology, 35(1): 96-101.

Klitzman, R. (2006), 'Post-residency disease and the medical self: Identity, work, and health care among doctors who become patients', Perspectives in Biology and Medicine, 49(4): 542-52.

Kunda, Z. (1990), 'The case for motivated reasoning', Psychological Bulletin, 108(3): 480-98.

Langelaan, M. (2017), Monitor Zorggerelateerde Schade 2015/2016: Dossieronderzoek bij overleden patiënten in Nederlandse ziekenhuizen. Utrecht: Nivel.

Leicht, K. T., T. Walter, I. Sainsaulieu and S. Davies (2009), 'New public management and new professionalism across nations and contexts', Current Sociology, 57(4): 581-605.

Linos, E., L. T. Quan and E. Kirkman (2020), 'Nudging early reduces administrative burden: Three field experiments to improve code enforcement', Journal of Policy Analysis and Management, 39(1): 243-65.

Lorenz-Spreen, P., S. Lewandowsky, C. R. Sunstein and R. Hertwig (2020), 'How behavioural sciences can promote truth, autonomy and democratic discourse online', Nature Human Behaviour, 4(11): 1102-1109.

Lusardi, A., A. S. Samek, A. Kapteyn, L. Glinert, A. Hung and A. Heinberg (2014), Visual tools and narratives: New ways to improve financial literacy. Global Financial Literacy Excellence Center Working Paper No. 2014-1; Becker Friedman Institute for Research in Economics Working Paper No. 2585231. Retrieved from: https://ssrn.com/abstract=2585231.

Marteau, T. M., D. Ogilvie, M. Roland, M. Suhrcke and M. P. Kelly (2011), 'Judging nudging: Can nudging improve population health?', British Medical Journal, 342: d228. doi:10.1136/bmj.d228.

Mikkelsen, M. F., C. B. Jacobsen and L. B. Andersen (2017), 'Managing employee motivation: Exploring the connections between managers' enforcement actions, employee perceptions, and employee intrinsic motivation', International Public Management Journal, 20(2): 183-205.

Mulderrig, J. (2017), 'Reframing obesity: A critical discourse analysis of the UK's first social marketing campaign', Critical Policy Studies, 11(4): 455-76.

Münscher, R., M. Vetter and T. Scheuerle (2016), 'A review and taxonomy of choice architecture techniques', Journal of Behavioral Decision Making, 29(5): 511-24.

Nagtegaal, R., L. Tummers, M. Noordegraaf and V. Bekkers (2019), 'Nudging healthcare professionals towards evidence-based medicine: A systematic scoping review', Journal of Behavioral Public Administration, 2(2). doi:10.30636/jbpa.22.71.

Neudecker, N., F. R. Esch, T. Schaefers and S. Valussi (2014), 'Message reframing in advertising', Psychology \& Marketing, 31(11): 946-57.

Nickerson, R. S. (1998), 'Confirmation bias: A ubiquitous phenomenon in many guises', Review of General Psychology, 2(2): 175-220.

Nisbett, R. E., G. T. Fong, D. R. Lehman and P. W. Cheng (1987), 'Teaching reasoning', Science, 238(4827): $625-31$. 
Noordegraaf, M. (2007), 'From "pure" to "hybrid" professionalism: Present-day professionalism in ambiguous public domains', Administration \& Society, 39(6): 761-85.

Noordegraaf, M. (2016), 'Reconfiguring professional work: Changing forms of professionalism in public services', Administration \& Society, 48(7): 783-810.

Noordegraaf, M. and B. Steijn (2014), Professionals under pressure: The reconfiguration of professional work in changing public services. Amsterdam: Amsterdam University Press.

Ölander, F. and J. Thøgersen (2014), 'Informing versus nudging in environmental policy', Journal of Consumer Policy, 37(3): 341-56.

Olsen, A. L. (2015), 'Citizen (dis)satisfaction: An experimental equivalence framing study', Public Administration Review, 75(3): 469-78.

Pittet, D., B. Allegranzi, H. Sax, S. Dharan, C. L. Pessoa-Silva, L. Donaldson and J. M. Boyce (2006), 'Evidence-based model for hand transmission during patient care and the role of improved practices', The Lancet Infectious Diseases, 6(10): 641-52.

Ramirez, G. and S. L. Beilock (2011), 'Writing about testing worries boosts exam performance in the classroom', Science, 331(6014): 211-3.

Sax, H., B. Allegranzi, M. N. Chraiti, J. Boyce, E. Larson and D. Pittet (2009), 'The World Health Organization hand hygiene observation method', American Journal of Infection Control, 37(10): 827-34.

Sedlmeier, P. and G. Gigerenzer (2001), 'Teaching Bayesian reasoning in less than two hours', Journal of Experimental Psychology: General, 130(3): 380-400. doi:10.1037/0096-3445.130.3.380.

Shadish, W. R., T. D. Cook and D. T. Campbell (2002), Experimental and quasi-experimental designs for generalized causal inference. Boston: Houghton Mifflin.

Squires, J. E., K. N. Suh, S. Linklater, N. Bruce, K. Gartke, I. D. Graham, A. Karovitch, J. Read, V. Roth, K. Stockton, E. Tibbo, K. Woodhal, J. Worthington and J. M. Grimshaw (2013), 'Improving physician hand hygiene compliance using behavioral theories: A study protocol', Implementation Science, 8(1): 16.

Sunstein, C. R. (2017), 'Nudges that fail', Behavioural Public Policy, 1(1): 4-25.

Sunstein, C. R. (2020), Behavioral science and public policy. Elements in Public Economics. Cambridge: Cambridge University Press.

Thaler, R. H. and C. R. Sunstein (2008), Nudge: Improving decisions about health, wealth, and happiness. New Haven: Yale University Press.

Tummers, L. (2019), 'Public policy and behavior change', Public Administration Review, 79(6): 925-30.

Tummers, L., V. Bekkers and B. Steijn (2009), 'Policy alienation of public professionals: Application in a new public management context', Public Management Review, 11(5): 685-706.

Tummers, L. L., V. Bekkers, E. Vink and M. Musheno (2015), 'Coping during public service delivery: A conceptualization and systematic review of the literature', Journal of Public Administration Research and Theory, 25(4): 1099-126.

Tversky, A. and D. Kahneman (1981), 'The framing of decisions and the psychology of choice', Science, 211 (4481): 453-8.

Van Roekel, H. (2020), Increasing compliance with the hospital hand hygiene protocol: Nudging or boosting?. Retrieved from: osf.io/hx4fu.

Weber, E. U. (2012), 'Doing the right thing willingly: Using the insights of behavioral decision research for better environmental decisions', in E. Shafir (ed.), The behavioral foundations of public policy, Princeton: Princeton University Press, 380-97.

Weske, U., P. Boselie, E. van Rensen and M. Schneider (2019), 'Physician compliance with quality and patient safety regulations: The role of perceived enforcement approaches and commitment', Health Services Management Research, 32(2): 103-12.

WHO (2009), WHO guidelines on hand hygiene in health care: A summary. Geneva: WHO.

Wilkinson, T. M. (2013), 'Nudging and manipulation', Political Studies, 61(2): 341-55.

Cite this article: van Roekel H, Reinhard J, Grimmelikhuijsen S (2022). Improving hand hygiene in hospitals: comparing the effect of a nudge and a boost on protocol compliance. Behavioural Public Policy 6, 52-74. https://doi.org/10.1017/bpp.2021.15 